\title{
Fresh Versus Frozen Testicular Sperm Samples in Microdissection Testicular Sperm Extraction Intracytoplasmic Sperm Injection Treatment
}

\section{Şafak HATIRNAZ1 ${ }^{1}$, Serdar BAŞARANOĞLU ${ }^{1}$, Ebru HATIRNAZ², Mine Kanat PEKTAŞ̧}

Istanbul,Turkey

\section{ABSTRACT}

OBJECTIVE: The present study aims to compare the clinical outcomes of fresh versus frozen testicular samples in patients with non-obstructive azoospermia who would undergo intracytoplasmic sperm injection procedure.

STUDY DESIGN: This is a retrospective review of 541 patients with non-obstructive azoospermia who consecutively underwent microdissection testicular sperm injection and intracytoplasmic sperm injection between January 2010 and October 2014.

RESULTS: A total of 4896 mature oocytes were collected from the partners of azoospermic men and 1894 sperms were retrieved by microdissection testicular sperm procedures. About 1036 fresh sperms were used to perform intracytoplasmic sperm injection in 296 men with non-obstructive azoospermia whereas 858 in 245 azoospermic men. Approximately 1228 embryos were obtained after intracytoplasmic sperm injection and 1080 embryos were transferred. After embryo transfer, 146 clinical pregnancies occurred and 125 pregnancies ended up with live birth. The fertilization, implantation, clinical pregnancy and live birth rates were respectively $44.6 \%, 33.4 \%, 28.0 \%$ and $24.7 \%$ for 296 fresh microdissection testicular sperm cycles. On the other hand, the fertilization, implantation, clinical pregnancy and live birth rates were respectively $46.5 \%, 32.7 \%, 25.7 \%$ and $21.2 \%$ for 245 frozen microdissection testicular sperm cycles. There was no statistically significant difference between the fresh and frozen microdissection testicular sperm injection cycles in aspect of fertilization, implantation, clinical pregnancy and liver birth rates $(p=0.125, p=0.194, p=0.196$ and $p=0.182)$.

CONCLUSION: The utilization of fresh and frozen sperms in microdissection testicular sperm - intracytoplasmic sperm injection cycles has similar clinical outcomes. The use of frozen sperms obtained by testicular sperm can be considered as an efficient and safe approach for avoiding unnecessary ovarian hyperstimulation and repetitious interventions on testicular tissues.

Keywords: Azoospermia, Intracytoplasmic sperm injection, In vitro fertilization, Microdissection testicular sperm extraction

Gynecol Obstet Reprod Med 2017;23(2):74-78

\section{Introduction}

Azoospermia is defined as the absence of any measurable level of sperm in ejaculate. Less than $1 \%$ of general population and 10 to $15 \%$ of infertile men are affected by azoosper-

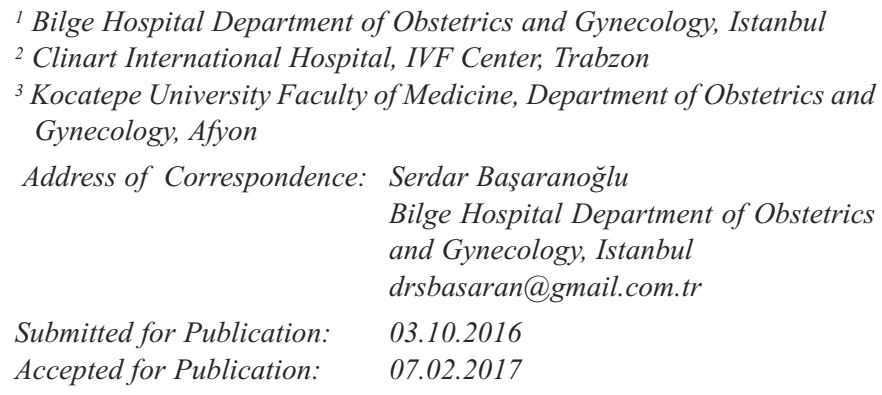

\begin{tabular}{|c|c|}
\hline & Access this article online \\
\hline $\begin{array}{c}\text { Quick Response Code: } \\
\text { 모․ }\end{array}$ & Website: www.gorm.com.tr \\
\cline { 2 - 3 } & DOI:10.201613/GORM.2016.636 \\
\hline
\end{tabular}

How to cite this article: Hatırnaz S. Başaranoğlu S. Hatırnaz E. Pektaș MK. Fresh Versus Frozen Testicular Sperm Samples in Microdissection Testicular Sperm Extraction Intracytoplasmic Sperm Injection Treatment. Gynecol Obstet Reprod Med 2017;23(2):74-8 mia. Obstruction of ductal system is responsible for approximately $40 \%$ of cases, but it may be related to non-obstructive causes in the remaining cases. Non-obstructive azoospermia is a clinical condition, which is associated with testicular failure. This condition comprises a spectrum of testicular pathologies resulting from various reasons that include chromosomal aberrations, congenital abnormalities, infectious agents, exposure to gonadotoxins, trauma, endocrine disorders, and idiopathic causes (1-4).

Men with azoospermia can father biological children by the retrieval of sperms from the male reproductive system for in vitro fertilization (IVF) / intracytoplasmic sperm injection (ICSI). An ideal surgical technique for this purpose should enable, with a minimal trauma to the testis, the retrieval of a sufficient amount of motile spermatozoa to inject all available oocytes and to cryopreserve the remainder, in case if a further attempt is needed $(5,6)$.

Microdissection testicular sperm extraction (MD TESE) is currently used for surgical retrieval of sperms. This technique combines the advantages of a less invasive approach with an 
open excisional biopsy, and thus, allows retrieval that is more efficient by identifying the zones of active spermatogenesis through optical magnification, together with minimal testicular trauma (7).

The efficiency and reliability of MD TESE have been verified by many clinical studies. It has been reported that sperm retrieval rates change between $35 \%$ and $77 \%$ for MD TESE procedures, and more importantly, MD TESE procedures have significantly better clinical outcomes than conventional TESE procedures or percutaneous aspirations (8-17).

The present study aims to compare the clinical outcomes of fresh versus frozen testicular samples in patients with nonobstructive azoospermia who would undergo intracytoplasmic sperm injection (ICSI) procedure.

\section{Material and Method}

This is a retrospective review of 541 patients with non-obstructive azoospermia who consecutively underwent MD TESE at the study center between January 2010 and October 2014. Urological evaluation, semen analysis and hormonal evaluation were performed for each patient. All patients gave written informed consent for MD TESE and ICSI procedures.

Microdissection Testicular Sperm Extraction: Testicular tissues of 541 azoospermic patients were removed by open testicular biopsy according to the technique previously described by Schlegel (7). All surgical procedures were performed by a single surgeon, under general anesthesia. Briefly, after stabilization of testicle, a small $2 \mathrm{~cm}$ transverse incision was made into the scrotal skin and carried through tunica vaginalis to expose tunica albuginea. After exploration through another $2 \mathrm{~cm}$ incision of the tunica albuginea that was parallel to the vessels and perpendicular to the epididymis, direct examination of the testicular parenchyma was carried out at $\mathrm{x} 5$ magnification under the operating microscope to identify individual seminiferous tubules that were larger, whiter, and typically more opaque than other tubules in the testicular parenchyma. Multiple small (3-4 mm) samples were excised sharply from larger and more opaque tubules. For each microdissected sample, the tissue around this area was then excised as a standard biopsy to evaluate the efficiency of sperm retrieval. Tissue samples were placed in sterile tubes containing IVF medium (Vitrolife G-IVFTM; Kungsbacka, Sweden) and transferred to andrology laboratory. No acute or chronic complications were observed in any of the patients.

Sperm Retrieval: In the andrology laboratory, tissue samples placed in Falcon tubes containing IVF medium were dispersed mechanically using two sterile glass slides immediately to obtain a suspension. The suspension was then examined under a phase-contrast microscope for the presence of spermatozoa. Surgery was terminated if spermatozoa were identified. If no spermatozoa were found after examination of the suspension, multiple samples from the second testis were taken. After identifying spermatozoa, the sperm suspension was transferred into sterile tubes and was centrifuged on a two-layer discontinuous Percoll gradient (50\% and 90\%) (PureSperm $\left.{ }^{\circledR} 100\right)$. The testicular tissue solution was incubated at $37^{\circ} \mathrm{C}$ and $6 \% \mathrm{CO}_{2}$ for 1 hour in a Petri dish. After the incubation, spermatozoa were collected by needle under an inverted microscope using x200 magnification and prepared for ICSI procedure.

The sperms were frozen on the day of retrieval. Testicular tissues were mixed 1:1 with sperm freezing medium (Vitrolife, Kungsbacka, Sweden) in cryo-vials and then kept at $-20^{\circ} \mathrm{C}$ for 20 minutes. After labeling, these samples were frozen by liquid nitrogen vapor technique. Thawing was performed at room temperature for 15 minutes. The preparation was then separated from the cryoprotectant by washing in culture medium and centrifugating at $2000 \mathrm{rpm}$ for 15 minutes. The resulting pellet was resuspended in $500 \mu \mathrm{l}$ of IVF culture medium.

Controlled Ovarian Hyperstimulation: Controlled ovarian hyperstimulation was carried out with mini-dose long-acting antagonist in 251 patients (130 patients in fresh MD-TESE cycles), standard dose long-acting gonadotropin antagonist in 213 patients (120 patients in fresh MD-TESE cycles), ultra mini-dose long-acting antagonist in 45 patients ( 25 patients in fresh MD-TESE cycles) and cc-gonadotropin antagonist in 32 patients (21 patients in fresh MD-TESE cycles). Antagonist based protocols were adopted for controlled ovarian hyperstimulation as they were referred to be short and simple regimens with good clinical outcomes and significant reduction in incidence of severe ovarian hyperstimulation syndrome (18).

Intracytoplasmic Sperm Injection Procedure and Embryo Transfer: All oocytes were inseminated by ICSI. Obtained spermatozoa were injected into metaphase II oocytes according to standard ICSI procedures. If oocyte collection and sperm retrieval were performed on the same day, fresh testicular sperm samples were used. The fertilized embryos were transferred into the uterine cavity 48-120 hours after the ICSI procedure using a Wallace catheter. The fertilization was verified by detection of two pronuclei within the oocytes 16-18 hours after microinjection. Clinical pregnancy was defined as a distinct intrauterine gestational sac visualized by transvaginal ultrasonography.

\section{Statistical Analysis}

Collected data were analyzed by Statistical Package for Social Sciences version 18.0 (SPSS IBM software, Armonk, NY, USA). Continuous variables were expressed as mean \pm standard deviation (range: minimum-maximum) and categorical variables were denoted as numbers or percentages where appropriate. Smirnov-Kolmogorov test was used to determine the distribution of variables. Student t-test, chi-square test, Mann-Whitney U test and Kruskal-Wallis test were used for the comparisons. Two-tailed p values less than 0.05 were accepted to be statistically significant. 


\section{Results}

This is a retrospective review of 541 men with non-obstructive azoospermia. Table 1 demonstrates the clinical characteristics of the reviewed MD TESE-ICSI cycles.

A total of 4896 mature oocytes were collected from the partners of azoospermic men and 1894 sperms were retrieved by MD-TESE procedures. About 1036 fresh sperms were used to perform ICSI in 296 men with non-obstructive azoospermia whereas 858 in 245 azoospermic men. Approximately 1228 embryos were obtained after ICSI and 1080 embryos were transferred. After embryo transfer, 146 clinical pregnancies occurred and 125 pregnancies ended up with live birth. There were 21 pregnancy losses including seven first trimester miscarriages and three perinatal deaths in the fresh MD TESEICSI group and nine first trimester miscarriages and two perinatal deaths in the frozen MD TESE-ICSI group.

Table 2 compares the clinical outcomes of fresh and frozen testicular sperm samples in men with non-obstructive azoospermia who underwent MD TESE-ICSI cycles. The azoospermic men who underwent ICSI treatment by using fresh and frozen sperms were statistically similar with respect to age, partner age, infertility duration, serum follicle stimulating hormone concentration, serum luteinizing hormone level, mature oocyte count per cycle, sperm count per cycle, transferred embryo number per cycle, fertilization rate, implantation rate, clinical pregnancy rate and live birth rate ( $p>0.05$ for all).

The implantation, clinical pregnancy and live birth rates were statistically similar with respect to ovulation induction protocols. The implantation rates were 33.8\%, 33.3\%, 32.0\% and $33.3 \%$ in the mini-dose long-acting antagonist, standard dose long-acting gonadotropin antagonist, ultra mini-dose long acting antagonist and cc-gonadotropin antagonist groups respectively $(\mathrm{p}=0.118)$. The clinical pregnancy rates were $29.2 \%$, $28.3 \%, 24.0 \%$ and $23.8 \%$ in the mini-dose long-acting antagonist, standard dose long-acting gonadotropin antagonist, ultra mini-dose long acting antagonist and cc-gonadotropin antagonist groups respectively $(\mathrm{p}=0.124)$. The live birth rates were $24.6 \%, 25 \%, 24 \%$ and $23.8 \%$ in the mini-dose long-acting antagonist, standard dose long-acting gonadotropin antagonist, ultra mini-dose long acting antagonist and cc-gonadotropin antagonist groups respectively $(\mathrm{p}=0.121)$.

Table 1: Clinical characteristics of the microdissection testicular sperm extraction-intracytoplasmic sperm injection cycles

\begin{tabular}{|l|c|}
\hline & Mean \pm Standard deviation (Minimum-Maximum) \\
\hline Male age (years) & $33.2 \pm 7.6(24-50)$ \\
Female age (years) & $28.5 \pm 6.9(22-40)$ \\
Infertility duration (years) & $8.5 \pm 3.2(2-19)$ \\
Follicle stimulating hormone $(\mathrm{mlU} / \mathrm{mL})$ & $12.38 \pm 6.14(1.02-56.45)$ \\
Luteinizing hormone $(\mathrm{mlU} / \mathrm{mL})$ & $9.45 \pm 3.60(1.06-38.49)$ \\
Sperm count per cycle $(106 / \mathrm{mL})$ & $3.54 \pm 1.18(1-5)$ \\
Mature oocyte count per cycle & $9.05 \pm 3.64(5-13)$ \\
Transferred embryos per cycle & $2.0 \pm 0.2(1-3)$ \\
Fertilization rate & $246(45.5 \%)$ \\
Implantation rate & $179(33.1 \%)$ \\
Clinical pregnancy rate & $146(27.0 \%)$ \\
Live birth rate & $125(23.1 \%)$ \\
\hline
\end{tabular}

Table 2: Clinical outcomes of the fresh and frozen sperm samples in microdissection testicular sperm extraction- intracytoplasmic sperm injection cycles

\begin{tabular}{|c|c|c|c|}
\hline & $\begin{array}{l}\text { Fresh MD TESE } \\
\qquad(\mathrm{n}=296)\end{array}$ & $\begin{array}{l}\text { Frozen MD TESE } \\
\qquad(n=245)\end{array}$ & $\mathrm{p}$ \\
\hline Male age (years) & $32.8 \pm 8.2$ & $34.1 \pm 7.2$ & 0.174 \\
\hline Female age (years) & $28.1 \pm 6.3$ & $29.0 \pm 7.5$ & 0.186 \\
\hline Infertility duration (years) & $9.0 \pm 3.2$ & $8.2 \pm 3.7$ & 0.258 \\
\hline Follicle stimulating hormone $(\mathrm{mlU} / \mathrm{mL})$ & $12.65 \pm 6.74$ & $12.05 \pm 5.83$ & 0.212 \\
\hline Luteinizing hormone (mIU/mL) & $9.59 \pm 3.82$ & $9.11 \pm 3.37$ & 0.244 \\
\hline Sperm count per cycle & $3.36 \pm 1.25$ & $3.84 \pm 1.04$ & 0.174 \\
\hline Mature oocyte count per cycle & $9.11 \pm 3.56$ & $9.00 \pm 3.72$ & 0.110 \\
\hline Transferred embryos per cycle & $1.9 \pm 0.4$ & $2.0 \pm 0.8$ & 0.162 \\
\hline Fertilization rate & $132(44.6 \%)$ & $114(46.5 \%)$ & 0.125 \\
\hline Implantation rate & $99(33.4 \%)$ & $80(32.7 \%)$ & 0.194 \\
\hline Clinical pregnancy rate & $83(28.0 \%)$ & $63(25.7 \%)$ & 0.196 \\
\hline Live birth rate & $73(24.7 \%)$ & $52(21.2 \%)$ & 0.182 \\
\hline
\end{tabular}




\section{Discussion}

Studies focusing on quantitative spermatogenesis have shown that there should be at least three mature spermatids in the cross-section of each seminiferous tubule so that spermatozoa could be detected within the ejaculate. Men with azoospermia usually have 0 to 3 mature spermatids in each seminiferous tubule, and this is the reason why sperms can be occasionally found in their ejaculates. Testicular spermatozoa can be retrieved successfully by the TESE procedure and used for ICSI in case of non-obstructive azoospermia. The success of TESE depends on the identification of seminiferous tubules containing spermatozoa. The MD TESE is a procedure, which provides success in approximately one-third of the azoospermic patients who previously failed sperm retrieval by other methods. This technique allows the direct visualization of larger, more opaque and whitish tubules which are presumed to contain germ cells with active spermatogenesis by an operative microscope. However, it is an invasive procedure with potential complications. Thus, strict criteria should be adopted in setting up an indication for MD TESE $(2,8,14,19)$.

Indeed, there are a number of studies, which focus on ICSI outcomes after sperm collection. Fertilization rates, implantation rates, clinical pregnancy and live birth rates are lower in couples with non-obstructive azoospermia than the couples with obstructive azoospermia and the couples with other causes of male infertility. In one report comparing the reproductive potential of azoospermic men undergoing sperm retrieval and ICSI, the likelihood of obtaining a live birth was 1.8-fold higher in men with obstructive azoospermia than in men with non-obstructive azoospermia. These findings indicate that men with non-obstructive azoospermia have decreased success potential in assisted reproduction. The major underlying reason for this reduction is the fact that testicular sperms of azoospermic men are more likely to have centriolar and genetic defects. Therefore, the ability of the sperms to fertilize the oocytes and induce the formation and development of a viable zygote is impaired $(9,20,21)$.

It has been shown that performing ICSI with fresh or frozen spermatozoa would produce similar results and that freezing does not affect spermatozoa (17). When sperm retrieval cannot be completed on the day of oocyte pick up for ICSI, the cryopreservation of oocytes would be offered to these couples. In these cases, the cryopreservation of testicular spermatozoa would help to avoid both unnecessary ovarian hyperstimulation and the need for repetitive biopsies for successive ICSI cycles. The spermatozoa could be frozen within the testicular tissues by means of minimal processing or extraction and purification. Freezing the intact tissue appears to be the best method as this allows better survival and motility rates after thawing $(22,23)$.

The utilization of frozen sperms retrieved by MD TESE in ICSI cycles yield fertilization rates changing from $44 \%$ to
$63 \%$, implantation rates altering between $28 \%$ and $36 \%$ and clinical pregnancy rate of $27 \%$ per embryo transfer. On the other hand, the use of fresh sperms collected by MD TESE in ICSI cycles results in fertilization rates differing between $37 \%$ and $69 \%$, implantation rates of $29 \%$ to $41 \%$ and clinical pregnancy rates ranging from $21 \%$ to $36 \%$ (24-28). Frozen testicular sperms may have possibly lower fertilization rates than the ejaculated or fresh epididymal sperms $(53.4 \%$ vs $64.8 \%$ or $58.5 \%$ respectively). This study has also demonstrated that the clinical pregnancy rates are $33.6 \%$ and $44.3 \%$ for the fresh and frozen sperms obtained by MD TESE in ICSI cycles (29). Another study has declared that sperms retrieved from fresh and frozen testicular tissues (44.7\% vs $46.7 \%$ ) can achieve similar fertilization rates. Moreover, no significant change could be noticed for the clinical pregnancy rates achieved by fresh and frozen testicular sperms (44.2\% vs 43.6\%) (30).

As for the present study, the fertilization, implantation, clinical pregnancy and live birth rates were $45.5 \%, 43.1 \%$, $37.0 \%$ and $33.1 \%$ respectively. The fertilization, implantation, clinical pregnancy and live birth rates were respectively $44.6 \%, 33.4 \%, 28.0 \%$ and $24.7 \%$ for 296 fresh MD-TESE cycles whereas the same rates were respectively $46.5 \%, 32.7 \%$, $25.7 \%$ and $21.2 \%$ for 245 frozen MD-TESE cycles. The findings of the present study suggest that the utilization of fresh and frozen sperms in MD TESE-ICSI cycles have similar clinical outcomes. Thus, use of frozen sperms obtained by TESE can be considered as an efficient and safe approach for avoiding unnecessary ovarian hyperstimulation and repetitious interventions on testicular tissues. The cryopreservation of sperm containing testicular tissues may be more feasible than the cryopreservation of sperms. In addition, performing ICSI before ovulation induction may provide more benefit in case of non-obstructive azoospermia $(29,30)$. However, the power of this study is limited by the relatively small cohort size, lack of correlation analysis and inconsistencies in sperm collection and storage methods. Further research is warranted to clarify the clinical outcomes of fresh and frozen sperms retrieved by MD TESE technique in ICSI cycles.

\section{References}

1. Dadhich P, Ramasamy R, Lipshultz LI. The male infertility office visit. Minerva Urol Nefrol 2015 Jun; 67(2):15768 .

2. Esteves SC, Miyaoka R, Agarwal A. An update on the clinical assessment of the infertile male. Clinics (Sao Paulo) 2011;66(4):691-700.

3. Wosnitzer M, Goldstein M, Hardy MP. Review of azoospermia. Spermatogenesis 2014 Mar 31;4:e28218.

4. Donoso P, Tournaye H, Devroey P. Which is the best sperm retrieval technique for non-obstructive azoospermia? A systematic review. Hum Reprod Update 2007 Nov-Dec;13(6):539-49. 
5. Ishikawa T. Surgical recovery of sperm in non-obstructive azoospermia. Asian J Androl 2012 Jan;14(1):109-15.

6. Lejeune H, Lapoirie M, Brosse A, Cuzin B, Giscard d'Estaing S, Plotton I. Medical optimisation of sperm retrieval in non obstructive azoospermia. Gynecol Obstet Fertil 2014 Sep;42(9):640-3.

7. Schlegel PN. Testicular sperm extraction: Microdissection improves sperm yield with minimal tissue excision. Hum Reprod 1999 Jan;14 (1):131-5.

8. Silber SJ. Microsurgical TESE and the distribution of spermatogenesis in non-obstructive azoospermia.Hum Reprod 2000 Nov;15(11):2278-84.

9. Esteves SC, Miyaoka R, Agarwal A. Sperm retrieval techniques for assisted reproduction. Int Braz J Urol 2011 SepOct;37(5):570-83.

10. Carpi A, Sabanegh E, Mechanick J. Controversies in the management of nonobstructive azoospermia.Fertil Steril 2009 Apr;91(4):963-70.

11. Ramasamy R, Ricci JA, Palermo GD, Gosden LV, Rosenwaks Z, Schlegel PN. Successful fertility treatment for Klinefelter's syndrome. J Urol 2009 Sep;182(3):1108-13.

12. Okada H, Dobashi M, Yamazaki T, Hara I, Fujisawa M, Arakawa S, et al. Conventional versus microdissection testicular sperm extraction for nonobstructive azoospermia. J Urol 2002 Sep;168(3):1063-7.

13. Amer M, Ateyah A, Hany R, Zohdy W. Prospective comparative study between microsurgical and conventional testicular sperm extraction in non-obstructive azoospermia: follow-up by serial ultrasound examinations. Hum Reprod 2000 Mar;15(3):653-6.

14. Tsujimura A. Microdissection testicular sperm extraction: Prediction, outcome, and complications. Int J Urol 2007 Oct;14(10):883-9.

15. El-Haggar S, Mostafa T, Abdel Nasser T, Hany R, Abgdel Hadi A. Fine needle aspiration vs. mTESE in non-obstructive azoospermia. Int J Androl 2008 Dec;31(6):595601.

16. Esteves SC, Prudencio C, Seol B, Verza S, Knoedler C, Agarwal A. Comparison of sperm retrieval and reproductive outcome in azoospermic men with testicular failure and obstructive azoospermia treated for infertility. Asian J Androl 2014 Jul-Aug;16(4):602-6.

17. Dafopoulos K, Griesinger G, Schultze-Mosgau A, Orief Y, Schöpper B, Nikolettos $\mathrm{N}$ et al. Cumulative pregnancy rate after ICSI with cryopreserved testicular tissue in nonobstructive azoospermia. Reprod Biomed Online 2005 Apr;10(4):461-6.

18. Toftager M, Bogstad J, Bryndorf T, Løssl K, Roskær J, Holland $\mathrm{T}$ et al. Risk of severe ovarian hyperstimulation syndrome in GnRH antagonist versus GnRH agonist protocol: RCT including 1050 first IVF/ICSI cycles. Hum Reprod 2016 Jun;31(6):1253-64.
19. Shahedi A, Khalili MA, Soleimani M, Morshedizad S. Ultrastructure of in vitro matured human oocytes. Iran Red Crescent Med J 2013 Dec;15(12):e7379.

20. Siristatidis C, Sergentanis TN, Vogiatzi P, Kanavidis P, Chrelias $\mathrm{C}$, Papantoniou $\mathrm{N}$ et al. In vitro maturation in women with vs. without polycystic ovarian syndrome: A systematic review and meta-analysis. PLoS One 2015 Aug;10(8):e0134696.

21. Xu YP, Xiang HF, Zou WW, Li ZL, Zhang ZG, Zhou P et al. Clinical application of in vitro maturation of human immature oocytes for infertile women with polycystic ovary syndrome. Zhonghua Fu Chan Ke Za Zhi 2012 Jan; 47(1):14-8.

22. Habermann H, Seo R, Cieslak J, Niederberger C, Prins GS, Ross L. In vitro fertilization outcomes after intracytoplasmic sperm injection with fresh or frozen-thawed testicular spermatozoa. Fertil Steril 2000 May;73(5): 955-60.

23. Küpker W, Schlegel PN, Al-Hasani S, Fornara P, Johannisson R, Sandmann J et al. Use of frozen thawed testicular sperm for intracytoplasmic sperm injection. Fertil Steril 2000 Mar;73(3):453-8.

24. Gil-Salom M, Romero J, Minguez Y, Rubio C, De los Santos MJ, Remohi J et al. Pregnancies after intracytoplasmic sperm injection with cryopreserved testicular spermatozoa. Hum Reprod 1996 Jun;11(6):1309-13.

25. Friedler S, Raziel A, Soffer Y, Strassburger D, Komarovsky D, Ronel R. Intracytoplasmic injection of fresh and cryopreserved testicular spermatozoa in patients with nonobstructive azoospermia-a comparative study. Fertil Steril 1997 Nov;68(5):892-7.

26. Oates RD, Mulhall J, Burgess C, Cunningham D, Carson R. Fertilization and pregnancy using intentionally cryopreserved testicular tissue as the sperm source for intracytoplasmic sperm injection in 10 men with non-obstructive azoospermia. Hum Reprod 1997 Apr;12(4):734-9.

27. Watkins W, Nieto F, Bourne H, Wutthiphan B, Speirs A, Baker HW. Testicular and epididymal sperm in a microinjection program: methods of retrieval and results. Fertil Steril 1997 Mar;67(3):527-35.

28. Devroey P, Liu J, Nagy Z, Gooessens A, Tournaye H, Camus $M$ et al. Pregnancies after testicular sperm extraction and intracytoplasmic sperm injection in non-obstructive azoospermia. Hum Reprod 1995 Jun;10(6):1457-60.

29. Van Steirteghem A, Nagy P, Joris H, Janssenswillen C, Staessen C, Verheyen G et al. Results of intracytoplasmic sperm injection with ejaculated, fresh and frozen-thawed epididymal and testicular spermatozoa. Hum Reprod 1998 Apr;13 Suppl 1:134-42.

30. Tavukcuoglu S, Al-Azawi T, Al-Hasani S, Khaki AA, Khaki A, Tasdemir S. Using fresh and frozen testicular sperm samples in couples undergoing ICSI-MicroTESE treatment. J Reprod Infertil 2013 Apr;14(2):79-84. 